\title{
The Relay Scheme over MAC for Data Transmission Performance in Railway Wireless Sensor Network
}

\author{
Sun-Hwa Lim, Hannah Kim, Young-il Kim, Won Ryu \\ Intelligent Convergence Media Research Division \\ Electronics and Telecommunications Research Institute (ETRI) \\ \{limsh, rtkim, yikim, wlyu\}@etri.re.kr
}

\begin{abstract}
Wireless sensor networks (WSNs) have been widely applied to a various areas. Especially in recent years, there has been an increasing interest in the adoption of emerging sensing technologies for a railway infrastructure monitoring. However, because the wireless communication of a railway is operated between sensor devices in poor environments such as high speed and much vibration, there may be a problem when the IEEE 802.15.4 and ZigBee would be applied in railway WSNs. Therefore, it is necessary a scheme to reduce data transmission delay and power consumption in railway WSNs. To solve this problem, the relay scheme over the MAC sublayer on the IEEE 802.15.4 standard is proposed in this paper. In this paper, the relay scheme could reduce data transmission delay and power consumption by about a half when data routing is performed on not the IP layer but the MAC sublayer.
\end{abstract}

Keywords—Wireless Sensor Network, IEEE 802.15.4, ZigBee, Railway, Relay

\section{INTRODUCTION}

Wireless sensor networks (WSNs) have been widely applied to habitat monitoring, healthcare, object tracking, battlefield surveillance, etc. Commonly, sensor devices are restricted in computation, storage, communication bandwidth, and, most importantly, energy supply [1]. Especially in recent years, there has been an increasing interest in the adoption of emerging sensing technologies for a railway infrastructure monitoring.

The IEEE 802.15.4 Task Group (TG4) [2], together with the ZigBee Alliance [3], has developed an entire communication protocol stack for Low-Rate Wireless Personal Area Networks (LR-WPAN) [4]. However, because the wireless communication of a railway is operated between sensor devices in poor environments such as high speed and much vibration, there may be a problem when IEEE 802.15.4 and ZigBee would be applied in railway WSNs. To transfer sensing data from sensor devices to a coordinator, data routing is generally processed on the IP layer of ZigBee. When a railway network topology is composed the number of hierarchical levels, data routing over the IP layer may be caused data transmission delay and more power consumption. Therefore, it is necessary a scheme to reduce data transmission delay and power consumption in railway WSNs.
To solve this problem, the relay scheme over the medium access control (MAC) sublayer on the IEEE 802.15.4 standard is proposed in this paper.

This paper is organized as follows. In section II, we briefly introduce IEEE 802.15.4 and ZigBee. In session III, we propose the relay scheme based IEEE 802.15.4 and ZigBee. In session IV, the performance of proposed scheme is analyzed. Finally, session V presents the conclusion.

\section{RELATED WORK}

\section{A. IEEE 802.15.4}

An IEEE 802.15.4 network may operate in any of three industrial, scientific, medical (ISM) frequency bands, presented in Table 1 . The $2.4 \mathrm{GHz}$ frequency band is the most potential for large-scale WSN applications, since the high radio data rate reduces the frame transmission time and thus the energy per transmitted and received bit. Also, network scalability is improved, since a higher number of nodes may communicate with each other within a given time period. In addition, the band is available in most countries worldwide. Hence, in this analysis we focus on the $2.4 \mathrm{GHz}$ band.

TABLE 1. IEEE 802.15.4 FREQUENCY BANDS AND DATA RATES

\begin{tabular}{|c|c|c|c|}
\hline Band & $\mathbf{8 6 8 M H z}$ & $\mathbf{9 1 5 M H z}$ & $\mathbf{2 . 4 G H z}$ \\
\hline Region & EU, Japan & US & Worldwide \\
\hline Channels & 1 & 10 & 16 \\
\hline Data rate & $20 \mathrm{kbps}$ & $40 \mathrm{kbps}$ & $250 \mathrm{kbps}$ \\
\hline
\end{tabular}

IEEE 802.15.4 defines three types of logical devices, a Personal Area Network (PAN) coordinator, a coordinator and a device. The PAN coordinator is the primary controller of PAN, which initiates the network and operates often as a gateway to other networks. Each PAN must have exactly one PAN coordinator. Coordinators collaborate with each other for executing data routing and network self-organization operations. Devices do not have data routing capability and can communicate only with coordinators. Due to the low performance requirements of devices, they may be implemented with very simple and low-cost platforms. The 
standard designates these low complexity platforms as Reduced Function Devices (RFD). Platforms with the complete set of MAC services are referred to as Full Function Devices (FFD) [5].

\section{B. ZigBee}

As WSNs are expected to assume a greater role in everyday life, ZigBee, the representative communication network protocol of WSN, has become an active research subject [6]. ZigBee positioning is considered a promising open standard that is used with low data rates and low power in costeffective wireless network products [7]. ZigBee complements the LR-WPAN standard with network and security layers, and application profiles. For security and reliability, ZigBee supports access control lists, packet freshness timers, and 128bit Advanced Encryption Standard (AES). Different stack profiles are defined for home control, building automation, and plant control applications. The first version of ZigBee specification was announced in December 2004 [5].

\section{III.PROPOSED RELAY SCHEME}

In this section, we propose an efficient relay scheme to overcome the problems of the above approach.

\section{A. Wireless Sensor Network and Protocol Stack}

Figure 1 shows a hierarchical wireless sensor network example. A sensor device shall associate through the (PAN) coordinator and send an alert message or measurement value to the (PAN) coordinator. A coordinator is responsible for initiating and maintaining the devices on the network. In tree networks, routers move data and control messages through the network using a hierarchical routing strategy. The PAN coordinator is the principal controller of an IEEE 802.15.4 2003-based network that is responsible for network formation The PAN coordinator must be a full function device (FFD).

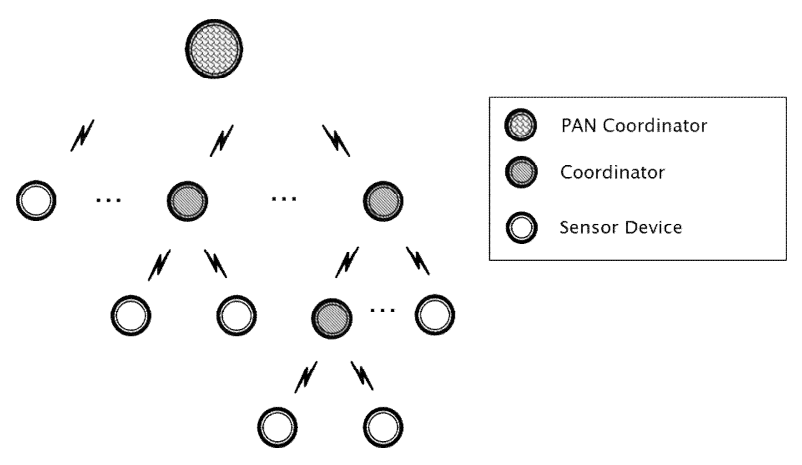

Figure 1. Hierarchy wireless sensor network example

Figure 2 shows protocol stack for data transmission between a sensor device and the PAN coordinator through a coordinator. Any data frame is transferred over the MAC sublayer of a coordinator to reduce data transmission delay and power consumption in railway WSNs.

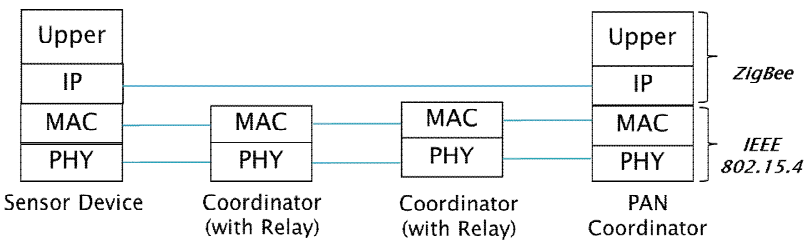

Figure 2. Protocol stack with relay of coordinator

\section{B. Newly Defined MAC Frame Structure}

For a device to be able to relay any data frame over the MAC sublayer to other device (e.g., a sensor device, a coordinator), it is necessary for us to modify the IEEE 802.15.4 MAC frame. The newly defined MAC frame is in Figure 3 . The relay address field is included in the addressing fields of the MAC header. The relay address is defined by two byes. The relay address shall specify the address of a next device to relay any data frame.

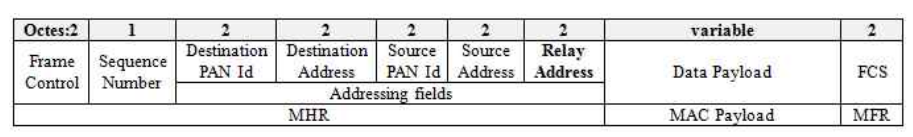

Figure 3. Newly defined MAC frame structure

\section{Relay- Enabled Device Procedure}

Figure 4 illustrates the procedure for relay function activation of the MAC sublayer. When the initialization for a device is operated, the higher layer transmits the MAC sublayer management entity (MLME)-RESET.request primitive with the RelaySupported parameter value of TRUE to the MAC sublayer. On receipt of this primitive, the relay function of the MAC sublayer is activated. The MAC sublayer shall send the MLME-RESET.confirm primitive in response to the MLME-RESET.request primitive to the higher layer.

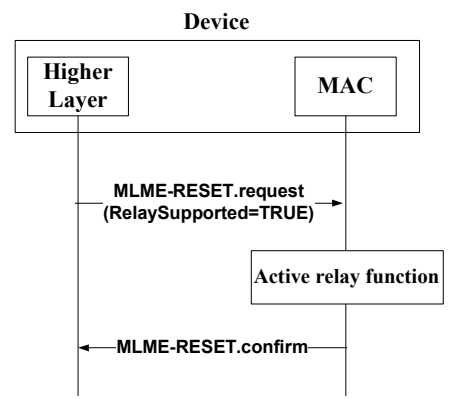

Figure 4. Procedure for relay function activation of the MAC sublayer

\section{Data Frame Transmission Procedure}

Figure 5 illustrates the procedure for data frame transmission. When the MAC sublayer of a sensor device receives the MAC common part sublayer (MCPS)DATA.request primitive with the destination address indicating the PAN coordinator, it shall research whether it has a valid a relay address in a relay table. If a valid relay address is found, a relay address is included in addressing fields of the MAC header. The MAC sublayer of a sensor device shall transmit any data frame to the MAC sublayer of a 
coordinator. A coordinator shall confirm its receipt by sending an acknowledgment frame. On receipt of this acknowledgment frame, the MAC sublayer shall send the $M C P S-D A T A . c o n f i r m$ primitive in response to the MCPS$D A T A$.request primitive to the higher layer.

The MAC sublayer of a coordinator shall research whether it has a valid relay address in a relay table if the received relay address equal to the MAC address of a coordinator. If found, a relay address is included in addressing fields of the MAC header. The MAC sublayer of a coordinator shall transmit any data frame to the MAC sublayer of the PAN coordinator. The PAN coordinator shall confirm its receipt by sending an acknowledgment frame. The PAN coordinator shall transmit the MCPS-DATA.indication primitive to the higher layer if the received relay address equal to the destination address of addressing fields. If not equal, the received data frame will be discarded.

\section{IV.Performance ANALYSis}

In this section, we analyze the performance comparison between the relay over MAC sublayer and IP routing over IP layer for any data frame transmission. In general, the higher layer of a device is developed on CPU, the MAC sublayer of a device is developed on Digital Signal Processing (DSP), and the PHY layer of a device is developed on field-programmable gate array (FPGA). Each layer can access dual-ported RAM (DPRAM) to read/write any data.

The (a) of Figure 6 illustrates IP routing over higher layer on a device and the procedure involves the following steps:

- The PHY layer shall write any MAC packet data unit (PDU) on DPRAM and interrupt to notify the MAC sublayer.

- The MAC sublayer shall read the MAC PDU from DPRAM, process the MAC PDU, and write the IP PDU on DPRAM.

- The higher layer shall poll the IP PDU from DPRAM, perform IP routing process, and write the IP PDU on DPRAM.

- The MAC sublayer shall poll the IP PDU from DPRAM, create the MAC PDU, write the MAC PDU on DPRAM, and interrupt to notify the PHY layer.

- The PHY layer shall read the MAC PDU from DPRAM.

The (b) of Figure 6 illustrates relay over the MAC sublayer on a device and the procedure involves the following steps:

- The PHY layer shall write any MAC PDU on DPRAM and interrupt to notify the MAC sublayer.

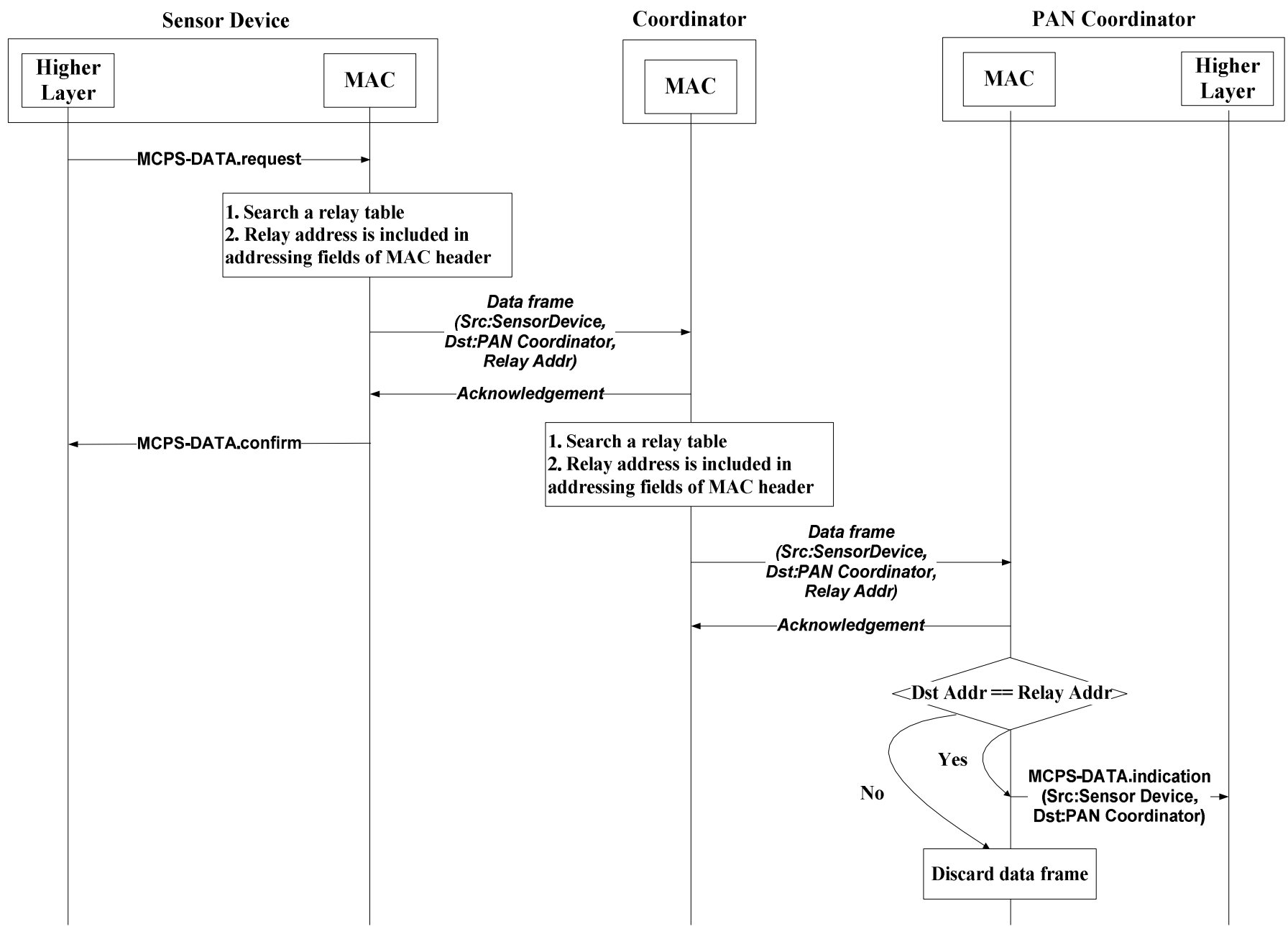

Figure 5. Procedure for data frame transmission 
- The MAC sublayer shall read the MAC PDU from DPRAM, process the MAC PDU to relay the MAC PDU, write the new MAC PDU on DPRAM, and interrupt to notify the PHY layer.

- The PHY layer shall read the MAC PDU from DPRAM.

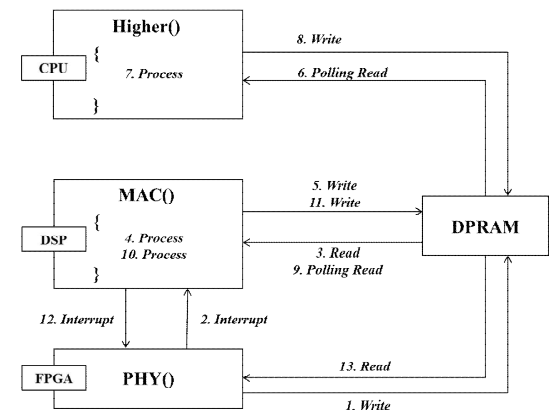

(a)

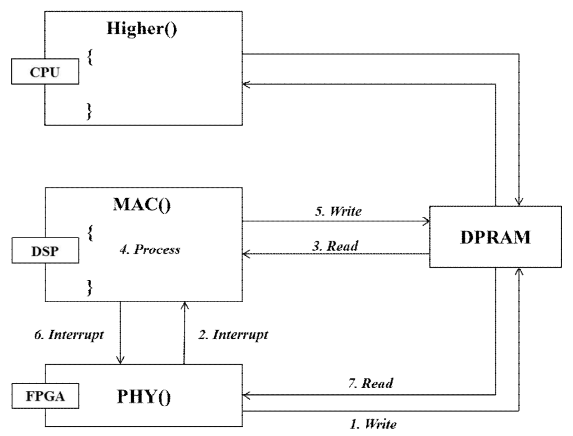

(b)

Figure 6. Steps for data frame process

Although IP routing procedure over higher layer is 13 steps, relay procedure over the MAC sublayer is 7 steps. The difference between IP routing and relay over the MAC sublayer is about $50 \%$. This may give us the reduction of data transmission delay and an expenditure of power.

\section{Conclusions}

In this paper, we presented the scheme to relay the MAC PDU over the MAC sublayer instead of the IP routing. The relay address in addressing fields of the MAC header was newly defined to relay the MAC PDU to other device. Additionally, we proposed the procedure for relay function activation of the MAC sublayer and the procedure for any data frame transmission. The proposed scheme can reduce data transmission delay and power consumption by about a half when data routing is performed on not the IP layer but the MAC sublayer.

\section{ACKNOWLEDGMENT}

This research is supported by the Convergence Research Business of Korea Research Council for Industrial Science \& Technology under B551179-12-07-00.

\section{REFERENCES}

[1] X. Yin, X. Zhou, R. Huang, and Y. Fang, "A fairness-Aware Congestion Control Scheme in Wireless Sensor Networks," IEEE
Transactions on Vehicular Technology, vol. 58, pp. 5225-5234, Nov. 2009.

[2] Part 15.4: Wireless MAC and PHY layer specifications for low-rate wireless personal area networks, IEEE Std 802.15.4, 2011.

[3] ZigBee Specifications, version 1.0, ZigBee Alliance, April 2005.

[4] A. Koubaa, M. Alves, and E. Tovar, "GTS Allocation Analysis in IEEE 802.15.4 for Real-Time Wireless Sensor Networks," Parallel and Distributed Processing Symposium, April 2006.

[5] M. Kohvakka, M. Kuorilehto, M. Hannikainen, and T. D. Hamalainen, "Performance Analysis of IEEE 802.15.4 and ZigBee for Large-Scale Wireless Sensor Network Applications,' in PE-WASUN'06, pp. 48-57, Oct. 2006.

[6] H. S. Kim, and J. Yoon, "Hybrid Distributed Stochastic Addressing Scheme for ZigBee/IEEE 802.15.4 Wireless Sensor Networks," ETRI Journal, vol. 33, pp. 704-711, Oct. 2011.

[7] B. S. Park, J. Y. Yoo, and H. B. Kim, "Modeling and Analysis of Queuing Effect of Two-Level Approach to Network Localization," ETRI Journal, vol. 34, pp. 625-628, Aug. 2012. 